Pacific Journal of Mathematic 


\section{ON THE BEHAVIOUR OF CAPILLARIES AT A CORNER}

\section{ERICH MieRSEMANN}

Consider the solution of the capillary surface equation over domains with a corner. It is assumed that the corner is bounded by lines. If the corner angle $2 \alpha$ satisfies $0<2 \alpha<\pi$ and $\alpha+\gamma<\pi / 2$ where $0 \leq \gamma<\pi / 2$ is the contact angle between the surface and the container wall then it is shown that the leading term which was discovered by Concus and Finn is equal to the solution up to $O\left(r^{\varepsilon}\right)$ for an $\varepsilon>0$ where $r$ denotes the distance from the corner.

We consider the non-parametric capillary problem in presence of gravity over a bounded base domain $\Omega \subset \mathbb{R}^{2}$ with a corner. That means, we seek a surface $S: u=u(x)$, defined over $\Omega$, such that $S$ meets vertical cylinder walls over the boundary $\partial \Omega$ in a prescribed constant angle $\gamma$ such that the following equations are satisfied, see Finn [3],

$$
\begin{gathered}
\operatorname{div} T u=\kappa u \text { in } \Omega, \\
\nu \cdot T u=\cos \gamma \quad \text { on the smooth parts of } \partial \Omega,
\end{gathered}
$$

where

$$
T u=\frac{D u}{\sqrt{1+|D u|^{2}}},
$$

$\kappa=$ const. $>0$ and $\nu$ is the exterior unit normal on $\partial \Omega$.

Let the origin $x=0$ be a corner of $\Omega$ with interior angle $2 \alpha$ satisfying

$$
0<2 \alpha<\pi \text {. }
$$

We assume that the corner is bounded by lines near $x=0$, see Figure 1. Furthermore, we assume that the contact angle satisfies

$$
0 \leq \gamma<\frac{\pi}{2} \text {. }
$$

Concus and Finn [2] have shown that $u$ is bounded near $x=0$ if and only if $\alpha+\gamma \geq \pi / 2$ is satisfied.

In the case $\alpha+\gamma>\pi / 2$ there exists an asymptotic expansion of $u$ near the origin, $\mathrm{cf}$. [4]. In the borderline case $\alpha+\gamma=\pi / 2$ Tam [5] 


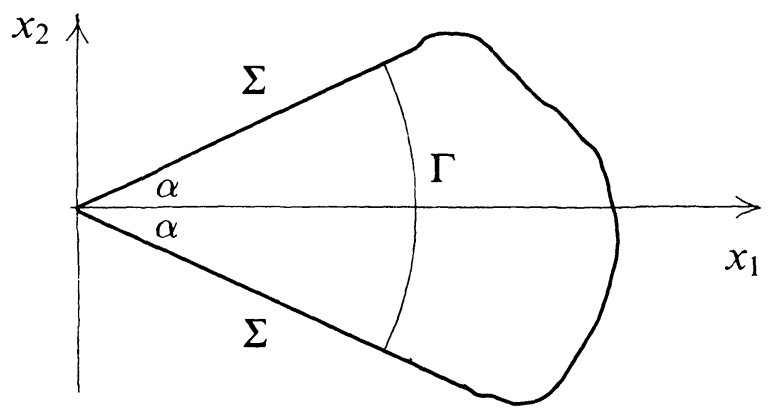

FIGURE 1

obtained that the normal vector to the surface $S$ is continuous up to the corner.

In this note we are interested in the case

$$
\alpha+\gamma<\frac{\pi}{2} \text {. }
$$

If (5) is satisfied, then a solution of (1), (2) is unbounded near the origin, see Concus and Finn [2] or Finn [3, Theorem 5.5]. Moreover, the leading term of a possible asymptotic expansion was given in these works. Here we show that this term is an approximation up to $O\left(r^{\varepsilon}\right)$ for the solution itself.

The easy proof is based on a comparison principle of Concus and Finn [1], see also Finn [3, Theorem 5.1] and requires only some calculations with barrier functions which are not much different from comparison functions used by Concus and Finn [2], see also Finn [3, Proof of Theorem 5.5].

Let $r, \theta$ be polar coordinates centered at $x=0$, and set $k=$ $\sin \alpha / \cos \gamma$.

THEOREM. Let $u$ be a solution of (1), (2). Then, provided (3), (4) and (5) are satisfied, one has for an $\varepsilon>0$ the expansion

$$
u=\frac{\cos \theta-\sqrt{k^{2}-\sin ^{2} \theta}}{k \kappa r}+O\left(r^{\varepsilon}\right)
$$

near the corner.

Proof. We set $B_{\rho}(0)=\left\{x \in \mathbb{R}^{2} ; x_{1}^{2}+x_{2}^{2}<\rho^{2}\right\}, \rho>0$, and $\Omega_{\rho}=$ $\Omega \cap B_{\rho}(0)$. Let

$$
v=\frac{\cos \theta-\sqrt{k^{2}-\sin ^{2} \theta}}{k \kappa r}
$$

and set $w=v-A r^{\lambda}$ where $A=$ const. $>0$ and $\lambda=$ const. $>0$. 
Using polar coordinates, we obtain after some calculation that there are positive numbers $r_{0}, K_{0}$ and $\lambda_{0}$ such that for all $r, A$ and $\lambda$ with

$$
0<r \leq r_{0}, \quad A>0, \quad 0<\lambda \leq \lambda_{0} \quad \text { and } \quad A \lambda \leq K_{0}
$$

we have

$$
\operatorname{div} T w=\kappa w+A \kappa r^{\lambda}+\eta_{1}+\eta_{2}
$$

in $\Omega_{r_{0}}$, where

$$
\left|\eta_{1}\right| \leq c_{1} r^{3} \text { and }\left|\eta_{2}\right| \leq c_{2} A \lambda r^{\lambda}
$$

The constants $c_{1}, c_{2}$ do not depend on $r, \lambda$ and $A$. Moreover, we find after calculation that

$$
\nu \cdot T w<\cos \gamma \quad \text { on } \Sigma_{r_{0}} \text { if } \gamma>0
$$

and

$$
\nu \cdot T w=\cos \gamma=1 \text { on } \Sigma_{r_{0}} \text { if } \gamma=0
$$

for all $r, A$ and $\lambda$ satisfying (6). Here we set

$$
\Sigma_{\rho}=\left[\partial \Omega \cap B_{\rho}(0)\right] \backslash\{0\} .
$$

Now, we choose $A_{1}$ and $\lambda_{1}\left(\lambda_{1}>0\right.$ small and $A_{1}>0$ large) from the region defined by $(6)$ such that

$$
A_{1}\left(\kappa-c_{2} \lambda_{1}\right) r^{\lambda_{1}}-c_{1} r^{3}>0
$$

is satisfied in $\Omega_{r_{1}}, r_{1}>0$ small enough, $r_{1} \leq r_{0}$. Set $\Gamma_{\rho}=\Omega \cap \partial B_{\rho}(0)$. We may choose $A_{2} \geq A_{1}$ and $\lambda_{2} \leq \lambda_{1}$ satisfying (6) such that we have $v\left(r_{1}, \theta\right)-A_{2} r_{1}^{\lambda_{2}}<u$ on $\Gamma_{r_{1}}$. The boundedness of $u$ on $\bar{\Gamma}_{r_{1}}$ is a consequence of a result of Concus and Finn [2], cf. also Finn [3, Proof of Theorem 5.5]. The inequality (10) remains valid for these $A_{2}, \lambda_{2}$ too. That means, we have obtained that $w=v-A_{2} r^{\lambda_{2}}$ satisfies $\operatorname{div} T w \geq \kappa w$ in $\Omega_{r_{1}}$, cf. (7) and (10), $\nu \cdot T w \leq \nu \cdot T u$ on $\Sigma_{r_{1}}$, cf. (8) or (9) and (2), and $w \leq u$ on $\Gamma_{r_{1}}$. The comparison principle of Concus and Finn, see for example Finn [3, Theorem 5.1], implies

$$
v-A_{2} r^{\lambda_{2}} \leq u \text { in } \Omega_{r_{1}} \text {. }
$$

Setting $w=v+A r^{\lambda}$, we obtain an upper bound for $u$ as follows. Again, by calculation we find in $\Omega_{r_{0}}$ for $r, A, \lambda$ satisfying (6) (we use the same notation for the constants $r_{0}, K_{0}, \ldots$, which may be different from the corresponding constants from above) that

$$
\operatorname{div} T w=\kappa w-A \kappa r^{\lambda}+\eta_{1}+\eta_{2}
$$


where $\eta_{1}, \eta_{2}$ fulfill the same inequalities as above. If $\gamma>0$, then for $r, A$ and $\lambda$ satisfying (6), we see after some calculation that

$$
\nu \cdot T w \geq \cos \gamma+c_{3} A \lambda r^{\lambda+1}-c_{4} r^{4}
$$

is true on $\Sigma_{r_{0}}$ with positive constants $c_{3}$ and $c_{4}$ not depending on $A, r$ and $\lambda$. Suppose that (6) and that for a positive constant $K_{1}, K_{1} \leq K_{0}$, the inequality $K_{1} \leq A \lambda$ is satisfied. In particular, we assume that

$$
K_{1} \leq A \lambda \leq K_{0}
$$

for $A$ and $\lambda$ from the region given by (6).

Now, inequality $\left(8^{\prime}\right)$ implies that there are positive constants $r_{1}, A_{1}$ and $\lambda_{1}$ such that one has

$$
\nu \cdot T w \geq \cos \gamma \quad \text { on } \Sigma_{r_{1}}
$$

for all $A$ and $\lambda$ with $A \geq A_{1}$ and $0<\lambda \leq \lambda_{1}$ satisfying (11). We may choose an $A=A_{2}$ and a $\lambda=\lambda_{2}$ such that the inequality

$$
-A_{2} r^{\lambda_{2}}\left(\kappa-c_{2} \lambda_{2}\right)+c_{1} r^{3}<0
$$

takes place in $\Omega_{r_{2}}$ for an $r_{2} \leq r_{1}$. Now, we take $A_{3}$ large enough, $A_{3} \geq A_{2}$, and $\lambda_{3}>0$ small enough, $\lambda_{3} \leq \lambda_{2}$, so that (11) and the next inequality (13) are both satisfied,

$$
v+A_{3} r_{2}^{\lambda_{3}} \geq u \text { on } \Gamma_{r_{2}} \text {. }
$$

Hence, since $\left(10^{\prime}\right)$ remains valid if $A_{2}$ is replaced by $A_{3}$ and $\lambda_{2}$ by $\lambda_{3}$, we obtain, see $\left(7^{\prime}\right)$ and $\left(10^{\prime}\right)$,

$$
\operatorname{div} T w \leq \kappa w \quad \text { in } \Omega_{r_{2}} .
$$

From (13), this inequality and because (12) is true on $\Sigma_{r_{2}}$ it follows

$$
v+A_{3} r^{\lambda_{3}} \geq u \text { in } \Omega_{r_{2}}
$$

from the comparison principle of Concus and Finn.

If $\gamma=0$, then the above considerations with respect to $\Sigma$ are superfluous since (9) takes place for this $w$ too. Thus, the theorem is proved.

REMARK. An inspection of the above proof shows that we have proven in fact a stronger result as formulated in the theorem: there exist positive constants $\rho_{0}, A$ and $\lambda$ only depending on $\alpha, \gamma$ and $\kappa$ and not on the particular solution $u$ considered such that

$$
|u-v| \leq A r^{\lambda} \quad \text { in } \Omega_{\rho_{0}} .
$$


This follows because $r_{1}$ in the above proof concerning the lower bound for $u$ and $r_{2}$ which occurs in the proof of the upper bound do not depend on $u$. Then we use that there are bounds for $|u|$ on $\bar{\Gamma}_{r_{1}}$ and $\bar{\Gamma}_{r_{2}}$ which do not depend on $u$ itself, compare Finn [3, Proof of Theorem 5.5].

\section{REFERENCES}

[1] P. Concus and R. Finn, On capillary free surfaces in the absence of gravity, Acta Math., 132 (1974), 177-198.

[2] _ On capillary free surfaces in a gravitational field, Acta Math., 132 (1974), 207-223.

[3] R. Finn, Equilibrium Capillary Surfaces, Springer, Berlin 1986.

[4] E. Miersemann, Asymptotic expansion at a corner for the capillary problem, Pacific J. Math., 134 (1988), 299-311.

[5] L.-F. Tam, Regularity of capillary surfaces over domains with corners: borderline case, Pacific J. Math., 125 (1986), 469-485.

Received May 15, 1988.

KARL-MARX UNIVERSITY

LEIPZIG, GDR 



\section{PACIFIC JOURNAL OF MATHEMATICS EDITORS}

\author{
V. S. VARADARAJAN \\ (Managing Editor) \\ University of California \\ Los Angeles, CA 90024-1555-05 \\ Herbert Clemens \\ University of Utah \\ Salt Lake City, UT 84112 \\ THOMAS ENRIGHT \\ University of California, San Diego \\ La Jolla, CA 92093
}

R. FINN

Stanford University

Stanford, CA 94305

HeRMANN FlaschKa

University of Arizona

Tucson, AZ 85721

VAUGHaN F. R. Jones

University of California

Berkeley, CA 94720

STEVEN KeRCKHOFF

Stanford University

Stanford, CA 94305

\author{
ROBION KIRBY \\ University of California \\ Berkeley, CA 94720 \\ C. C. MOORE \\ University of California \\ Berkeley, CA 94720 \\ HAROLD STARK \\ University of California, San Diego \\ La Jolla, CA 92093
}

\section{ASSOCIATE EDITORS}
R. ARENS
E. F. BECKENBACH
B. H. NEUMANN
F. WOLF
K. YoshidA (1906-1982)

\section{SUPPORTING INSTITUTIONS}

UNIVERSITY OF ARIZONA

UNIVERSITY OF BRITISH COLUMBIA

CALIFORNIA INSTITUTE OF TECHNOLOGY

UNIVERSITY OF CALIFORNIA

MONTANA STATE UNIVERSITY

UNIVERSITY OF NEVADA, RENO

NEW MEXICO STATE UNIVERSITY

OREGON STATE UNIVERSITY
UNIVERSITY OF OREGON

UNIVERSITY OF SOUTHERN CALIFORNIA

STANFORD UNIVERSITY

UNIVERSITY OF HAWAII

UNIVERSITY OF TOKYO

UNIVERSITY OF UTAH

WASHINGTON STATE UNIVERSITY

UNIVERSITY OF WASHINGTON 


\section{Pacific Journal of Mathematics}

Vol. 140, No. $1 \quad$ September, 1989

Michel Brestovski, Algebraic independence of solutions of differential

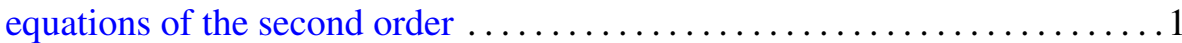

Bohumil Cenkl, Cohomology operations from higher products in the de

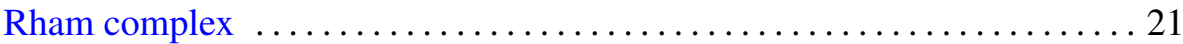

Gustavo Corach and Daniel Suarez, Generalized rational convexity in

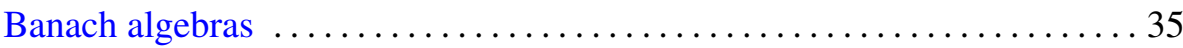

Keresztély Corrádi and Sándor Szabó, A new proof of Rédei’s theorem . . . 53

Steven R. Costenoble and Stefan Waner, Equivariant orientations and

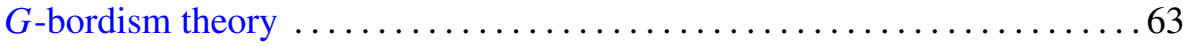

Angel Granja, Apéry basis and polar invariants of plane curve

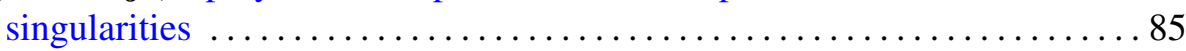

Young Soo Jo, Isometries of tridiagonal algebras .................. 97

Ronald Leslie Lipsman, Harmonic analysis on exponential solvable homogeneous spaces: the algebraic or symmetric cases $\ldots \ldots \ldots \ldots \ldots 117$

Erich Miersemann, On the behaviour of capillaries at a corner 149

Marian Nowak, On the finest Lebesgue topology on the space of essentially

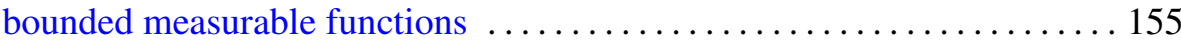

Pascal J. Thomas, Hardy interpolating sequences of hyperplanes ........ 163

H. Bevan Thompson, Differentiability properties of subfunctions for second order ordinary differential equations 